\title{
Estenosis de la colostomía secundaria a colitis actínica tardía. A propósito de un caso
}

\author{
Colostomy stenosis due to chronic radiation enteritis. Report of clinical case
}

Estíbaliz Echazarreta-Gallego ${ }^{*}$, Manuela Elía-Guedea1, Elena Córdoba-Díaz de Laspra, Ariel G. Gonzales-Sejas ${ }^{2}$, Alba Hernáez-Arzoz ${ }^{1}$ y José M. Ramírez-Rodríguez ${ }^{1}$

'Servicio de Cirugía General y del Aparato Digestivo, Hospital Clínico Universitario de Zaragoza, Instituto de Investigación Sanitaria Aragón (IIS Aragón); ${ }^{2}$ Servicio de Anatomía Patológica, Hospital Clínico Universitario de Zaragoza. Zaragoza, España

\section{Resumen}

Antecedentes: La colitis actínica es una endarteritis obliterante ocasionada por exposición a radiación ionizante. Se manifiesta de manera aguda (primeras semanas) o crónica (hasta 30 años después). Caso clínico: Paciente tratado por neoplasia rectal mediante quimiorradioterapia y amputación abdominoperineal. En su estudio por cuadros oclusivos se objetiva una estenosis colónica desde la colostomía hasta el ángulo hepático. Se lleva a cabo cirugía resectiva, con resultado favorable. Conclusiones: En los próximos años es previsible un aumento en la incidencia de esta patología. Resultará fundamental establecer un nivel de sospecha que nos permita detectar los casos, así como establecer medidas que prevengan su aparición.

Palabras clave: Cáncer colorrectal. Colitis actínica. Radioterapia.

\begin{abstract}
Background: Radiation colitis is a obliterans endarteritis caused by exposure to ionizing radiation. It manifests early or late in time. Case report: We present a case of rectal cancer treated with chemoradiotherapy and abdominoperineal resection ten years before. Patient suffered occlusive periods and colonoscopy showed stenotic colon extending from the colostomy up hepatic flexure. Colonic resection surgery was performed. Conclusions: It will be essential to establish a high level of suspicion that allows us to detect cases and it also will be essential to establish measures to prevent its occurrence.
\end{abstract}

Key words: Colorectal cancer. Radiation colitis. Radiation.

\section{Antecedentes}

La colitis actínica o enteritis por radiación es una alteración de la mucosa colorrectal secundaria a radioterapia abdominal o pélvica. Fue descrita por primera vez por Walsh en $1897^{1}$ tras una exposición accidental abdominal a radiación ionizante, y posteriormente ha sido recogida por otros autores ${ }^{2}$. La lesión anatomopatológica producida es una endarteritis obliterante progresiva que ocasiona atrofia de la mucosa, fibrosis y neoangiogénesis ${ }^{3}$. Se han descrito una forma aguda, desarrollada durante las primeras 8 semanas tras la exposición a la radiación, y una

\section{Correspondencia:}

*Estíbaliz Echazarreta-Gallego

Avda. San Juan Bosco, 15

C.P. 50009, Zaragoza, España

Cir Cir. 2020;88(S1):48-50

E-mail: esti.egallego@hotmail.com 
crónica, observada entre 2 meses y hasta 30 años después ${ }^{4}$. La forma crónica puede presentar las siguientes manifestaciones clínicas: rectorragia, malabsorción, diarrea, tenesmo, perforación, fístula o dolor ${ }^{5}$. Presentamos un caso de manifestación tardía (9 años) que requirió tratamiento quirúrgico para su resolución.

\section{Caso clínico}

Paciente de 66 años con antecedentes de adenocarcinoma de tercio inferior del recto (T3NOMO) tratado con quimiorradioterapia neoadyuvante y amputación abdominoperineal en 2005. Controles oncológicos sin evidencia de recidiva neoplásica (última tomografía computarizada, analítica sanguínea incluyendo marcadores tumorales [CEA-Ca 19.9] y colonoscopia de control realizadas en 2011, sin alteraciones). El paciente se mantiene asintomático desde la intervención hasta 2014, momento en que presenta un episodio de suboclusión secundaria a una estenosis localizada a nivel de la colostomía, manejada satisfactoriamente mediante dilataciones endoscópicas. Seis meses después, el paciente consulta por un episodio de diarreas de varias semanas de evolución. Se realiza un estudio con colonoscopia que objetiva estenosis de la luz colónica que se extiende desde la colostomía hasta $14 \mathrm{~cm}$, donde la estenosis hace infranqueable el paso del endoscopio y de los dilatadores por un alto riesgo de perforación (Fig. 1). Se plantea tratamiento quirúrgico y se completa el estudio mediante enema opaco, que muestra una reducción segmentaria del calibre intestinal hasta el ángulo esplénico. En diciembre de 2014 se interviene de manera programada y se objetiva la estenosis colónica, que se continúa más allá de lo esperado por las pruebas complementarias efectuadas, hasta el ángulo hepático. Se realiza resección del segmento afecto (desde el cabo colostómico al ángulo hepático colónico) y nueva colostomía en la fosa ilíaca derecha. El paciente evoluciona de manera favorable, sin recidiva del proceso oclusivo. La anatomía patológica (Fig. 2) informó de enteritis actínica sin signos neoplásicos.

\section{Discusión}

La radioterapia es una parte fundamental en el tratamiento de diversas neoplasias abdomino-pélvicas. Sin embargo, a pesar del progreso reciente en cuanto a planificación de campos, sus efectos secundarios

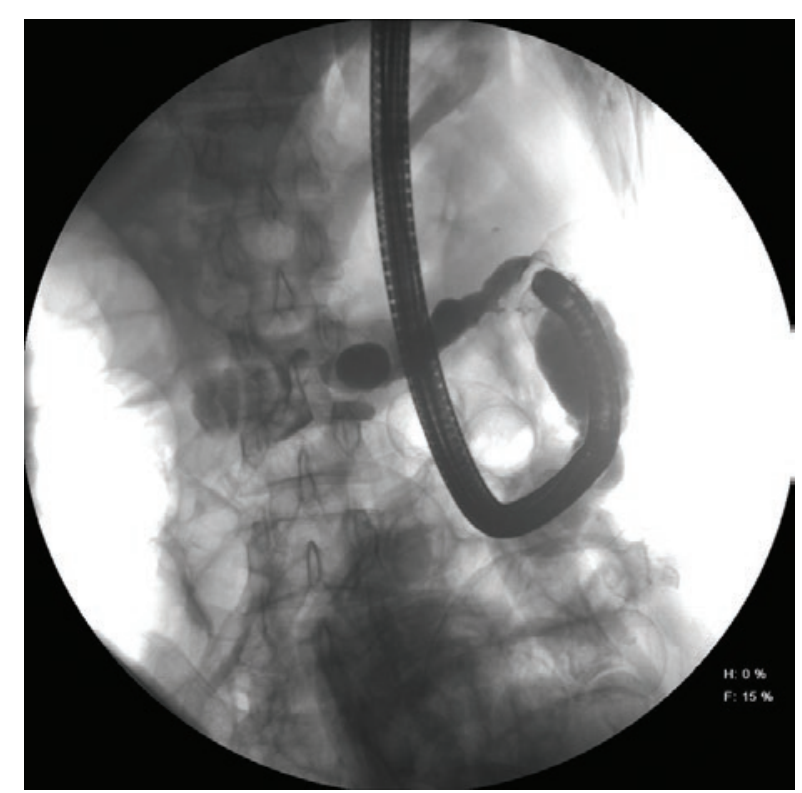

Figura 1. Radiografía tras endoscopia con administración de contraste que muestra estenosis colónicas.

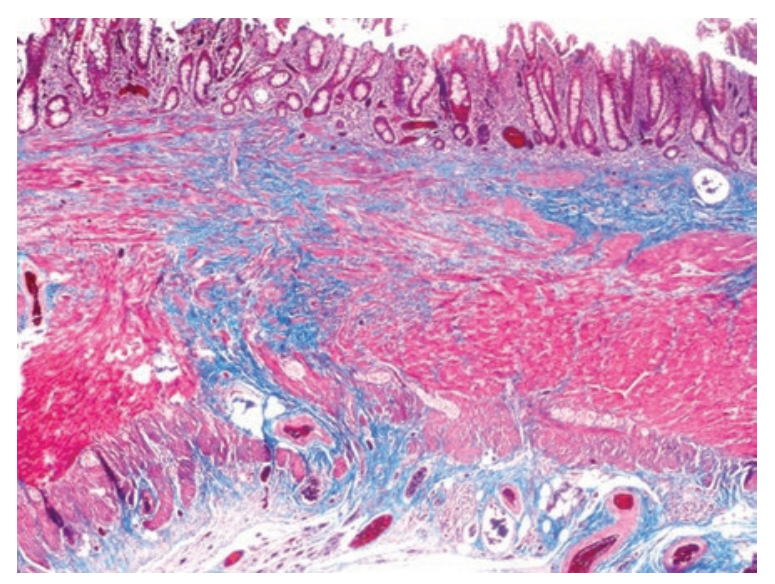

Figura 2. Anatomía patológica. Tricrómico de Masson que pone de manifiesto el depósito de fibras de colágeno en color azul (fibrosis transmural), acentuado en la submucosa.

continúan siendo un problema clínico importante; hasta el $80 \%$ de los pacientes que reciben radioterapia pélvica presentarán alteraciones en el hábito defecatorio, de las cuales un $20 \%$ tendrán un impacto moderado-intenso en la calidad de vida 6 . Esto está influido por ser la dosis de radiación terapéutica efectiva (40-50 Gy) de gran número de neoplasias próxima a la dosis de tolerancia de las células del intestino delgado y del colon ${ }^{7}$. La enteritis crónica secundaria a radioterapia es dependiente de la dosis y se estima entre el 0.5 y el $25 \%$, aunque esta cifra puede estar infraestimada por la inespecificidad de los síntomas. 
Son conocidos algunos factores que incrementan la incidencia, como la combinación de radioterapia interna y externa, asociada a quimioterapia y cirugía abdominopélvica previa, sexo femenino, edad avanzada y vasculopatía, algunos de ellos presentes en el caso. Con el fin de prevenir estas complicaciones se han descrito agentes radioprotectores que excluyen el intestino del campo de radiación ${ }^{8}$.

El diagnóstico clínico de sospecha se apoya en la realización de una rectoscopia o colonoscopia que mostrará una mucosa friable con neovascularización y permitirá la toma de biopsias (anatomopatológicamente son característicos los depósitos de fibroblastos y colágeno en la submucosa con endarteritis obliterante en la capa endotelial). Las técnicas de imagen pueden resultar de utilidad; la enterorresonancia mostrará cambios focales (engrosamiento de la pared intestinal, hiperrealce de la mucosa, entre otros) $)^{9}$, especialmente en el íleon, y en los casos en que se sospeche estenosis podría resultar de utilidad el estudio radiológico con contraste (enema opaco).

El tratamiento de la colitis actínica se establecerá en función de las características de los síntomas y de su intensidad. Cuando el sangrado sea el síntoma predominante, e intenso o persistente, se tratara por vía oral con antibióticos y antiinflamatorios (metronidazol, 5-aminosalicilatos), o por vía rectal mediante enemas (sucralfato, hidrocortisona, formaldehído, 5 -aminosalicilatos, ácidos grasos de cadena corta) ${ }^{10}$, o por vía endoscópica (argón-plasma, aplicación tópica de formalina, láser) con intención de coagular focos hemorrágicos activos ${ }^{11}$. La terapia con oxígeno hiperbárico, que actúa favoreciendo el recambio celular, ha demostrado ser de utilidad en la mejoría de los síntomas ${ }^{12}$. Alrededor de un tercio de los pacientes que presenten colitis actínica van a precisar cirugía durante el seguimiento, indicada ante la presencia de fístulas, estenosis o sangrado incoercible. El papel de la cirugía en la obstrucción intestinal crónica es controvertido por la alta morbimortalidad que implica. Se recomienda establecer un programa de soporte nutricional previo a la cirugía, por la alta tasa de malnutrición que presentan estos pacientes ${ }^{13}$.

\section{Conclusiones}

En los próximos años es esperable un aumento de la incidencia de colitis actínica secundaria al empleo de tratamientos radioterápicos. Un diagnóstico de sospecha será fundamental para establecer tratamientos que mitiguen la sintomatología, pero es prioritario orientar los esfuerzos hacia la prevención de la aparición de esta patología mediante una planificación radioterápica adecuada.

\section{Conflicto de intereses}

Los autores declaran no tener ningún conflicto de intereses.

\section{Responsabilidades éticas}

Protección de personas y animales. Los autores declaran que para esta investigación no se han realizado experimentos en seres humanos ni en animales.

Confidencialidad de los datos. Los autores declaran que han seguido los protocolos de su centro de trabajo sobre la publicación de datos de pacientes.

Derecho a la privacidad y consentimiento informado. Los autores han obtenido el consentimiento informado de los pacientes y/o sujetos referidos en el artículo. Este documento obra en poder del autor de correspondencia.

\section{Bibliografía}

1. Walsh D. Deep tissue traumatism from Roentgen ray exposure. BMJ. 1897;2:272-3.

2. Martínez Hernández Magro P. Oclusión intestinal secundaria a enteritis por radiación. Reporte de caso. Rev Gastroenterol Mex 2015;80:111-3.

3. Andreyev J. Gastrointestinal complications of pelvic radiotherapy: are they of any importance? Gut. 2005;54:1051-4.

4. Tagkalidis PP, Tjandra JJ. Chronic radiation proctitis. ANZ J Surg. 2001;71:230-7.

5. González GJL, Menéndez NJ, Ponce SR. Actinic colon stenosis after 22 years. Revista Cubana de Cirugía. 2014;53:303-8.

6. Andreyev J. Gastrointestinal symptoms after pelvic radiotherapy: a new understanding to improve management of symptomatic patients. Lancet Oncol. 2007;8:1007-17.

7. Yoshimura K, Hirata I, Maemura K, Sugi K, Tahara T. Radiation enteritis: a rare complication of the transverse colon in uterine cancer. Intern Med. 2000;39:1060-3.

8. Harb AH, Abou Fadel C, Sharara Al. Radiation enteritis. Curr Gastroenterol Rep. 2014;16:383.

9. Algin O, Turkbey B, Ozmen E, Algin E. Magnetic resonance enterography findings of chronic radiation enteritis. Cancer Imaging. 2011;11:189-94.

10. Grodsky MB, Sidani SM. Radiation proctopathy. Clin Colon Rectal Surg. 2015;28:131-41.

11. Vanneste BGL, Van De Voorde L, de Ridder RJ, Van Limbergen EJ, Lambin P, van Lin EN. Chronic radiation proctitis: tricks to prevent and treat. Int J Colorectal Dis. 2015;30:1293-303.

12. Bennett MH, Feldmeier J, Hampson NB, Smee R, Milross C. Hyperbaric oxygen therapy for late radiation tissue injury. Cochrane Database Syst Rev. 2016;(4):CD005005.

13. Webb GJ, Brooke R, De Silva AN. Chronic radiation enteritis and malnutrition. J Dig Dis. 2013;14:350-7. 